\title{
Análise longitudinal do desempenho dos estudantes de Ensino Médio e estudantes de nível superior para Algoritmos e Programação
}

\author{
Maria Claudete Schorr ${ }^{1}$, Magda Bercht ${ }^{1}$ \\ ${ }^{1}$ Programa de Pós Graduação de Informática na Educação - PPGIE \\ Universidade Federal do Rio Grande do Sul (UFRGS) - Porto Alegre - RS - Brasil \\ bercht@inf.ufrgs.br, mclaudetesw@univates.br
}

\begin{abstract}
This paper shows a study that investigates the Basic Educational disciplines that contributes for development of competencies and skills for teaching Algorithm and Programming on Higher Education. An analysis of means of 137 students was performed among disciplines of High School and the Algorithm and Programming discipline of a Higher Educational Institution. The outcomes show that students with better performance in disciplines such as: Mathematics, Physics and Portuguese acquire skills and knowledge that support them to development questions of logical reasoning and problem solving required on the programming disciplines.
\end{abstract}

Resumo. Este artigo apresenta um estudo que investiga as disciplinas do ensino médio que podem contribuir para o desenvolvimento das competências e habilidades necessárias para a aprendizagem de Algoritmos e Programação do Ensino Superior. Fez-se uma análise das médias de cada disciplina do Ensino Médio e da disciplina de Algoritmos e Programação de 137 alunos de uma IES. Os resultados demonstram que alunos com melhor desempenho nas disciplinas de Matemática, Física e Português adquirem competências e conhecimentos que lhes dão suporte para o desenvolvimento de questões de raciocínio lógico e resolução de problemas exigidos nas disciplinas de programação.

\section{Introdução}

O presente artigo apresenta um estudo longitudinal de estudantes do Ensino Médio frente as suas médias na disciplina de Algoritmos e Programação nos cursos da área da Computação. Verifica-se que se os estudantes apresentam dificuldades na aprendizagem dos conteúdos em programação podem estar relacionadas a dificuldades da Educação Básica.

Graminha em 1994 já afirmava o que se pode verificar em Fraga e Gonçalves (2017), que a dificuldade de aprendizagem por parte dos estudantes da Educação Básica para realizar a interpretação de enunciados de situações problemas e o desenvolvimento de soluções por meio de algoritmos está relacionada com aspectos emocionais e comportamentais. Segundo Marturano et. al. (1993) e Borges et. al. (2013) as dificuldades apresentadas são múltiplas, de caráter afetivo-sociais e cognitivos. Estas dificuldades são percebidas nas disciplinas vinculadas à área das Ciências Exatas, como 
VII Congresso Brasileiro de Informática na Educação (CBIE 2018)

Anais dos Workshops do VII Congresso Brasileiro de Informática na Educação (WCBIE 2018)

Matemática, Física e Química do Ensino Médio, onde se faz necessário compreender o problema, extrair dele os dados, aplicar em fórmulas específicas para produzir o resultado esperado, estabelecendo assim um raciocínio lógico estruturado em forma de algoritmo.

No Ensino Superior, existem, de maneira análoga, dificuldades no processo de aprendizagem de Algoritmos e Programação. Segundo APA (2014) e Silva et. al. (2017) as habilidades acadêmicas fundamentais, tais como: leitura e escrita, interpretação e cálculos e raciocínio matemático devem ser desenvolvidas no período de escolarização formal dos estudantes, ou seja, na Educação Básica. Especificamente a disciplina de Algoritmos e Programação de computadores dos cursos de Computação, segundo Hoed (2016), vem sendo alvo de discussões em congressos, fóruns e estudos científicos, devido ao grande número de evasões e reprovações apresentadas nesta disciplina. Segundo Amaral (2015) os estudantes são incapazes de desenvolver o raciocínio lógico para propor soluções algorítmicas, assim como para abstração necessária na compreensão do problema. Neste sentido Amaral (2015) após pesquisar 25 IES (Instituições de Ensino Superior), propôs uma nova versão da disciplina sem alterar a carga horária, inserindo o uso de ferramentas com blocos visuais e programação para mundos virtuais. Com isso, segundo o autor os estudantes tiveram mais facilidade para desenvolver as soluções dos problemas propostos.

Brackmann (2017), Koscianski e Glizt (2017), Henrique e Tedesco (2017) e Hoed (2016) demonstram preocupação com a aprendizagem no que diz respeito à resolução de problemas, abstração, desenvolvimento de soluções algorítmicas. Neste sentido Brackmann (2017) propôs desenvolver as habilidades do Pensamento Computacional (PC) na Educação Básica, já nas séries iniciais, onde foram realizadas atividades desplugadas (sem a utilização do computador). Henrique e Tedesco (2017) realizaram uma RSL (Revisão Sistemática de Literatura) investigando as habilidades, competências, conhecimento e atitudes necessárias para a aprendizagem de algoritmos. Hoed (2016) realizou um estudo investigando as causas da evasão na disciplina de Algoritmos e Koscianski e Glizt (2017) visando melhorar as habilidades nos estudantes, desenvolveram atividades lúdicas para o raciocínio lógico na Educação Básica.

Para Wing (2006, p. 33) PC envolve conceitos de solução de problemas, projeto de sistemas, e entendimento do comportamento humano, desenhando os conceitos fundamentais da ciência da computação. Segundo Selby e Woollard (2012) apud Araújo et al. (2016 p. 1148) "PC é um processo cognitivo que reflete as habilidades de pensar de forma abstrata e algorítmica, saber decompor atividades complexas, bem como realizar avaliações e generalizações".

A pesquisa, em relato, tem como objetivo apresentar as disciplinas da Educação Básica que contribuem para o desenvolvimento das habilidades e competências necessárias para a aprendizagem de Algoritmos e Programação do Ensino Superior. Neste sentido, mapeou-se o desempenho dos estudantes no Ensino Superior, mais especificamente na disciplina de Algoritmos e Programação e seu desempenho durante os três últimos anos do Ensino Médio. Como resultados são apresentadas análises que demonstram a relação existente do desempenho desses estudantes traçados em uma linha longitudinal do tempo desde o Ensino Médio até a Graduação (disciplina de Algoritmos). 
VII Congresso Brasileiro de Informática na Educação (CBIE 2018)

Anais dos Workshops do VII Congresso Brasileiro de Informática na Educação (WCBIE 2018)

O artigo a partir daqui está organizado da seguinte forma: na seção 2 apresentase o referencial teórico, na seção 3 é apresentada a metodologia; na 4 são apresentados os resultados; na seção 5 são apresentadas as conclusões e trabalhos futuros.

\section{Referencial Teórico}

\subsection{Resolução de problemas}

No intuito de desafiar o estudante a se envolver em uma tarefa onde o método a ser utilizado para encontrar a solução ainda desconhecida, significa estar utilizando a resolução de problemas como metodologia de ensino (ROMANATTO, 2012). Segundo o autor, ao utilizar esta metodologia, conceitos e princípios podem ser melhor compreendidos pelos estudantes, tendo em vista que esta forma ativa preocupa-se em adquirir, elaborar e investigar, tornando sua aprendizagem significativa.

Romanatto (2012) ressalta a importância da representação do problema por meio de esquemas, diagramas ou mesmo desenhos representativos. Estes, permitem ao estudante estruturar suas ideias, estruturar a sequência e os recursos que serão utilizados para chegar na solução do problema. Polya (1995), por outro lado, define que a estruturação por meio de etapas representa um aspecto fundamental no processo de resolução de problemas, apresentando quatro etapas sendo elas: entender o problema; construir uma estratégia de resolução; executar a estratégia e revisar a solução.

As disciplinas de Algoritmos e Programação são fundamentais para os cursos da área da Computação. Estudos empíricos apontam que os estudantes do nível superior chegam despreparados, demonstrando não ter habilidade de resolução de problemas (HENRIQUE; TEDESCO, 2017). Uma das preocupações da Sociedade Brasileira de Computação (SBC) conforme Oliveira et. al. (2014) está no ensino de programação onde as habilidades fundamentais para a programação já deveriam ser desenvolvidas na Educação Básica.

\subsection{Cenário atual das Universidades em relação ao ensino de Algoritmos e Programação}

No cenário atual os cursos da área da Computação estão perdendo alunos pelo fato destes se sentirem desmotivados e despreparados para a aprendizagem do conteúdo proposto nas disciplinas de Algoritmos e Programação. Sentido a preocupação em mudar a forma de ensinar Algoritmos e Programação vem sendo constante. Em sua pesquisa Filho et. al. (2007) apresenta que a evasão anual nas IES principalmente públicas, têm variado entre 9 e $15 \%$ no período de 2000 a 2005 , neste mesmo período as IES privadas apresentaram uma oscilação próxima de 26\%. Conforme Hoed (2016, p. 17), a SEMESP (Sindicato das Entidades Mantenedoras de Estabelecimentos de Ensino Superior no Estado de São Paulo) realizou uma pesquisa concluindo que: "a cada três alunos que entram no curso de Sistemas de Informação, apenas um recebe o diploma. Em Ciência da Computação, a cada quatro alunos que entram no curso, apenas um termina".

A preocupação com a aprendizagem de Algoritmos e Programação é constante, por esse motivo, trabalhos científicos estão sendo realizados com aplicações de metodologias ativas (LIMA et. al., 2016); softwares com ambientes visuais como o 
VII Congresso Brasileiro de Informática na Educação (CBIE 2018)

Anais dos Workshops do VII Congresso Brasileiro de Informática na Educação (WCBIE 2018)

Scratch e a robótica (CARDOSO \&ANTONELLO, 2015); e a iniciativa de ensinar computação já na educação básica para desenvolver habilidades e competências que possam auxiliar na aprendizagem diminuindo as reprovações e evasões por meio de atividades do PC (BRACKMANN, 2017).

\subsection{Habilidades e competências}

O termo competência segundo Behar (2013, p.21), “ ...é compreendido de acordo com o ponto de vista do indivíduo, ou seja, como a reunião ou o conjunto de condições, recursos, elementos disponíveis aplicados em determinada situação". $\mathrm{Na}$ área da educação passou a ser utilizada no século XX, incialmente na Educação Profissional e mais tarde na educação em geral. Ainda conforme a autora a competência não é formada apenas por habilidades ou conhecimentos, mas sim, por habilidades, conhecimento e atitudes. Perrenoud (1999) afirma que ter competência significa ter (Conhecimentos, Habilidades e Atitudes ou CHA).

Conforme Perrenoud (2001), na competência o conhecimento é o saber do indivíduo. Behar (2013) menciona que muitas vezes habilidade é vista como sinônimo de competência, porém para Perrenoud (2000), habilidade faz parte da estrutura da competência, se mostrando menos ampla do que a competência. De acordo com Behar (2013, p. 28), "Habilidade é uma ação automatizada, um procedimento já construído, algo da ordem do operacional, não exigindo se deter em uma reflexão mais aprofundada".

Para Perrenoud (1999) o sujeito quando resolve uma situação-problema da vida real, sem ao menos pensar ou planejar, está usando uma habilidade. Ainda segundo o autor, habilidade é uma série de procedimentos mentais que o indivíduo aciona para resolver uma situação real, onde ele precise tomar uma decisão. Por exemplo, quando um aluno está aprendendo a multiplicar ele utiliza a habilidade da adição e da conservação do número, que ele já possui, para resolver o novo problema.

\subsection{Trabalhos relacionados}

A maioria dos estudos sobre o ensino e aprendizagem e programação tem como objetivo desenvolver ou utilizar ferramentas computacionais que apoiam este ensino (SILVA, et. al., 2015). No entanto para Silva, et. al. existe uma carência de trabalhos que investiguem os estudantes de nível básico, mencionando a existência de possibilidades de se trabalhar programação nas escolas.

Aspectos referentes ao ensino de computação que segundo os autores precisam ser investigados: "aprendizado independente ou em sincronia com assuntos vistos em sala de aula; uso de diferentes abordagens pedagógicas; habilidades e competências que podem ser exploradas; ensino presencial ou à distância; dentre outros". Em sua RSL foram analisadas publicações em revistas e congressos renomados na área: Revista Brasileira de Informática na Educação (RBIE); Revista Novas Tecnologias na Educação (RENOTE); Workshop de Informática na Escola (WIE); Workshop de Educação em Computação (WEI); Simpósio Brasileiro de Informática na Educação (SBIE); Simpósio Brasileiro de Jogos e Entretenimento Digital (SBGAMES).

Nesta abordagem 73 estudos foram considerados relevantes para análise destes $63 \%$ são estudos voltados às Universidades, 34\% na Educação Básica e 3\% foram realizados na Educação Básica e Universidade, segundo os autores isto demonstra que 
VII Congresso Brasileiro de Informática na Educação (CBIE 2018)

Anais dos Workshops do VII Congresso Brasileiro de Informática na Educação (WCBIE 2018)

mais estudos devem ser realizados na Educação Básica. Quanto às disciplinas da Educação Básica que estão relacionadas à programação apenas 5 estudos foram realizados, destes 2 trabalhos apresentam a Matemática, Física, Português, Química e Geografia apenas um trabalho apresenta cada disciplina. Em relação às habilidades e competências que estão sendo exploradas nos alunos, os autores em sua análise encontraram habilidades cognitivas, sociais e emocionais, nestes 29 estudos apresentaram as habilidades cognitivas de "resolver problemas, estabelecer conclusões lógicas e planejar e tomar decisões". Nas habilidades sociais aparece em destaque em 5 trabalhos "lidar com regras", "cooperar e elaborar". Como habilidades emocionais, são apresentadas "autoconfiança, autoestima e autoavaliação" em apenas um trabalho. Quanto às competências, estas foram citadas em apenas dois estudos, sendo elas: "criatividade, estruturação do pensamento, responsabilidade, curiosidade, confiança e trabalho em equipe". Ao concluírem a RSL os autores identificaram que a principal dificuldade relatada pelos estudantes está na compreensão do problema e no raciocínio lógico.

Henrique e Tedesco (2017), analisaram 26 artigos apresentados no SBIE (2012 à 2016) e na WalgProg (2015 à 2016) com objetivo investigar os conhecimentos, atitudes, habilidades e competências desejáveis para a aprendizagem de algoritmos. Após a análise os resultados apontaram que existem diversos conhecimentos, habilidades, atitudes e competências desejáveis para que ocorra a aprendizagem de algoritmos e programação. No que se referem às habilidades os autores encontraram como mais citadas: "Raciocínio lógico, saber estruturar o programa, reconhecer padrões de resolução de problemas do mundo real" e quanto às competências: "Criatividade, analisar a própria performance e abstração".

Delgado et. al (2004) desenvolveu uma metodologia para a aprendizagem de algoritmos e programação dividida em 3 fases: "Primeira Fase - Resolução de Problemas, nesta são utilizadas atividades lúdicas como quebra-cabeças, jogos, charadas, entre outras atividades de diferentes domínios; Segunda fase - Formalização, esta etapa é dividida em duas fases, onde na primeira o estudante formaliza uma solução na linguagem natural e na segunda é realizada a conscientização de dos diferentes níveis de linguagem; Terceira Fase - Construção de Algoritmos, nesta fase ocorre o refinamento das soluções e utilização de procedimentos sistemáticos" (DELGADO, 2004, p.5).

Esta metodologia pró-ativa foi aplicada em turmas iniciais dos cursos da área da computação no Ensino Superior, com o objetivo de melhorar o desempenho dos estudantes as atividades utilizadas durante as três fases incentivam o desenvolvimento do raciocínio lógico, da capacidade de abstração e solução de problemas, bem como, a autonomia cognitiva do estudante. Como resultado da proposta os autores mencionam a importância de mais testes, visto que a metodologia está no seu primeiro estágio. Em 2005 Delgado et. al. aplicaram a proposta para uma possível identificação de competências associadas a aprendizagem de leitura e construção de algoritmos. Nesta pesquisa foi realizado um mapeamento das subcompetências contidas nas competências de leitura e construção de algoritmos por meio das dificuldades e facilidades apresentadas pelos estudantes durante a aplicação da metodologia proposta por Delgado et. al. em 2004. Foram identificadas sub competências Técnico-Profissionais e competências Sociais e Comportamentais. 
VII Congresso Brasileiro de Informática na Educação (CBIE 2018)

Anais dos Workshops do VII Congresso Brasileiro de Informática na Educação (WCBIE 2018)

Diante deste cenário sentiu-se a necessidade de mapear as disciplinas do Ensino Médio que estão diretamente ligadas ao desenvolvimento de habilidades e competências necessárias para a aprendizagem de Algoritmos e Programação do Ensino Superior.

\section{Metodologia}

A presente pesquisa é de caráter exploratório, onde segundo Chemin (2015, p. 57), "É utilizada nas situações em que se exige um estudo exploratório para um conhecimento mais profundo do problema da pesquisa". Neste trabalho buscou-se investigar a relação existente entre o desempenho obtido pelos estudantes na disciplina de Algoritmos e Programação com o seu desempenho no Ensino Médio. Para tanto foram coletadas as respectivas médias das disciplinas de Português, Literatura, Artes, Biologia, História, Geografia, Ed. Física, Filosofia, Sociologia, Inglês, Matemática, Física e Química dos 3 anos do Ensino Médio. A pesquisa caracteriza-se também como quantitativa pelo fato de ter sido realizada uma análise dessas médias objetivando avaliar o desempenho desses estudantes. Conforme Malhotra apud Chemin (2015, p. 57), "a pesquisa quantitativa tem como objetivo quantificar os dados e generalizar os resultados da amostra para a população-alvo".

Foram analisadas as notas de 137 estudantes, ingressantes na disciplina Algoritmos e Programação no primeiro e segundo semestre de 2016 e no primeiro semestre de 2017, pertencendo aos cursos de Sistemas de Informação, Engenharia de Software, Engenharia da Computação e Redes de Computadores. Os dados foram classificados em três categorias conforme resultados obtidos em seu primeiro ingresso na disciplina, visto que, alguns desistiram ou reprovaram, logo, realizaram mais de uma matrícula na disciplina. As categorias foram criadas com base na média para aprovação da IES, sendo igual ou maior a 6. As categorias analisadas foram: Aprovados, Reprovados e Desistentes.

A coleta dos dados foi realizada diretamente no Arquivo Central da IES e os dados tabulados por meio de uma planilha eletrônica. Nesse processo foram registradas as médias de cada disciplina do Ensino Médio juntamente a média obtida na disciplina de Algoritmos e Programação, respeitando as categorias referentes à situação ao término da mesma. Foram contabilizados 22 Reprovados, 32 Desistentes e 83 Aprovados.

\section{Resultados e discussões}

A análise dos resultados foi realizada cruzando as notas de desempenho dos estudantes de Algoritmos da IES com as suas respectivas médias do Ensino Médio, tendo em vista avaliar o nível de influência do desempenho deste estudante no EM para o acompanhamento do conteúdo da disciplina de Algoritmos e Programação.

Dos 137 estudantes analisados, 73 são oriundos de escolas estaduais de EM, 23 de escolas estaduais de Ensino Politécnico, 28 de escolas particulares, 12 de Institutos Federais e 1 de EJA. Analisando a distribuição dos estudantes, percebe-se que em todas as categorias (Aprovados, Reprovados e Desistentes) existem estudantes destes segmentos, porém é perceptível (Figura 1) que no grupo dos estudantes Aprovados existe um percentual maior de alunos que oriundos de escolas particulares e IFs. 
VII Congresso Brasileiro de Informática na Educação (CBIE 2018)

Anais dos Workshops do VII Congresso Brasileiro de Informática na Educação (WCBIE 2018)

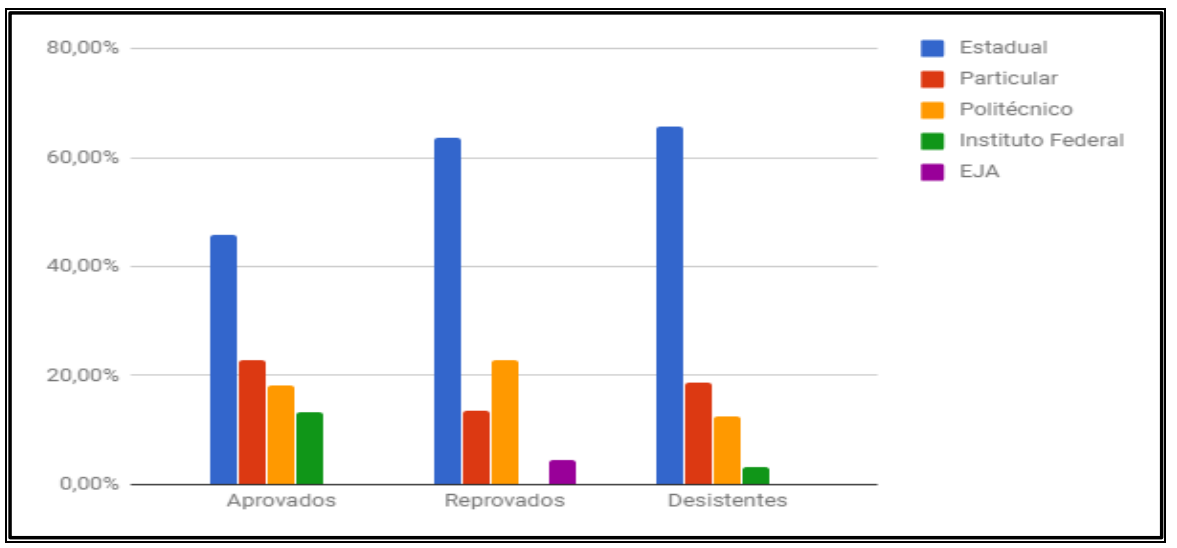

Figura 1. População analisada de acordo com o tipo de instituição de ensino

Ao analisar o desempenho dos estudantes na disciplina de Algoritmos e Programação, os estudantes Aprovados obtiveram média 8,45, os Reprovados 2,2 e os Desistentes não possuem nota pelo fato de não terem concluído a disciplina. Olhando especificamente a categoria dos Aprovados, os estudantes dos IFs (11 estudantes) apresentam média geral 9,23, os estudantes oriundos de escolas Particulares (19 estudantes) 8,36, do Ensino Médio Politécnico 8,5 e das escolas Estaduais 8,24. Tendo em vista que os estudantes dos IFs já possuem disciplinas voltadas à área de programação, justifica-se a facilidade na disciplina de Algoritmos pelo fato dos mesmos já possuírem conhecimentos prévios sobre o assunto. Dentre os demais tipos de instituição de ensino, a diferença na média varia apenas 3\% (entre 8,24 e 8,5), não apresentando variação significativa.

Os Reprovados na disciplina de Algoritmos e Programação das escolas Particulares (3) apresentam média geral 3,16, os estudantes do Ensino Médio Politécnico (4) 1,07, das escolas Estaduais (14) 2,37 e o estudante (1) do EJA ficou com média 1,3. Nesta categoria não teve nenhum estudante do IF. A diferença das médias entre os estudantes das categorias Aprovados e Reprovados é significativa, demonstrando que estes não tiveram a mesma formação e dedicação na Educação Básica. Além de coletar e registrar as notas de Algoritmos e Programação, fez-se o levantamento das médias dos estudantes nos 3 anos do Ensino Médio das disciplinas de Educação Física, Artes, Matemática, Física, Português, Sociologia, Inglês, Filosofia, Geografia, Química, História, Biologia e Literatura. Estas médias foram retiradas do histórico escolar de cada estudante, dispostas em um quadro (Quadro 1) e organizadas lado a lado nas categorias Aprovado, Reprovado e Desistentes. Em seguida, foi calculada a média de cada disciplina em cada uma das categorias e gerada uma classificação ordenada de forma decrescente.

Analisando a classificação das disciplinas sob o ponto de vista dos Aprovados, observa-se que as cinco primeiras colocações dessa categoria estão dispostas de acordo com sua área de conhecimento, da seguinte forma: $1^{\circ}$ Ciências da Saúde (Educação Física), $2^{\circ}$ e $5^{\circ}$ Linguística, Letras e Artes (Artes e Português), e $3^{\circ}$ e $4^{\circ}$ Ciências Exatas e da Terra (Matemática e Física). Essas mesmas disciplinas aparecem, respectivamente, na categoria Reprovados nas colocações: $1^{\circ}, 5^{\circ}, 10^{\circ}, 12^{\circ}$ e $13^{\circ}$. As disciplinas de Artes e Educação Física ainda aparecem na parte superior da listagem entre as cinco primeiras colocações, no entanto, as disciplinas de Matemática, Português e Física passaram a 
VII Congresso Brasileiro de Informática na Educação (CBIE 2018)

Anais dos Workshops do VII Congresso Brasileiro de Informática na Educação (WCBIE 2018)

ocupar a parte inferior da classificação. Traçando uma relação com a disciplina de Algoritmos e Programação que tem seu foco voltado para o desenvolvimento de soluções algorítmicas, estabelece-se uma linha de similaridade no que tange a lógica de resolução de problemas, fortemente trabalhada nas disciplinas da Educação Básica de Matemática e Física. Já a disciplina de Português que faz uso da interpretação, imaginação e organização de ideias durante o processo de construção do conhecimento, assemelha-se aos passos realizados na concepção de soluções algorítmicas, pois, para o entendimento do problema e projeção da solução, faz-se necessário compreender, interpretar e organizar as ideias a respeito do assunto em questão.

Quadro 1: Médias dos estudantes no Ensino Médio

\begin{tabular}{|c|c|c|c|c|c|c|c|c|}
\hline Disciplina & Aprovados & Classificação & Disciplina & Reprovados & Classificação & Disciplina & Desistentes & Classificação \\
\hline ED. Física & 8,22 & $1^{\circ}$ & ED. Física & 7,56 & $1^{\circ}$ & ED. Física & 8,28 & $1^{\circ}$ \\
\hline Artes & 8 & $2^{\circ}$ & Sociologia & 7,37 & $2^{\circ}$ & Artes & 7,66 & $2^{\circ}$ \\
\hline Matemática & 7,84 & $3^{\circ}$ & Filosofia & 7,22 & $3^{\circ}$ & Sociologia & 7,58 & $3^{\circ}$ \\
\hline Física & 7,57 & $4^{\circ}$ & Geografia & 6,9 & $4^{\circ}$ & Filosofia & 7,3 & $4^{\circ}$ \\
\hline Português & 7,54 & $5^{\circ}$ & Artes & 6,78 & $5^{\circ}$ & Geografia & 7,08 & $5^{\circ}$ \\
\hline Sociologia & 7,53 & $6^{\circ}$ & Inglês & 6,65 & $6^{\circ}$ & História & 6,91 & $6^{\circ}$ \\
\hline Inglês & 7,52 & $7^{\circ}$ & Literatura & 6,53 & $7^{\circ}$ & Inglês & 6,87 & $7^{\circ}$ \\
\hline Filosofia & 7,5 & $8^{\circ}$ & História & 6,17 & $8^{\circ}$ & Química & 6,76 & $8^{\circ}$ \\
\hline Geografia & 7,4 & $9^{\circ}$ & Química & 6,09 & $9^{\circ}$ & Literatura & 6,76 & $9^{\circ}$ \\
\hline Química & 7,37 & $10^{\circ}$ & Matemática & 6 & $10^{\circ}$ & Física & 6,71 & $10^{\circ}$ \\
\hline História & 7,22 & $11^{\circ}$ & Biologia & 6 & $11^{\circ}$ & Matemática & 6,65 & $11^{\circ}$ \\
\hline Biologia & 7,09 & $12^{\circ}$ & Português & 5,99 & $12^{\circ}$ & Português & 6,48 & $12^{\circ}$ \\
\hline Literatura & 7,08 & $13^{\circ}$ & Física & 5,98 & $13^{\circ}$ & Biologia & 6,41 & $13^{\circ}$ \\
\hline
\end{tabular}

Com base nos dados analisados foi possível identificar que os estudantes da categoria Aprovados tiveram melhores resultados nas disciplinas de Matemática, Física e Português em relação aos estudantes da categoria Reprovados. Quando comparada a média geral dessas disciplinas observou-se que as mesmas apresentam superioridade de $30 \%$, 26\% e 26,7\%, respectivamente. Os estudantes Reprovados apresentam nessas disciplinas notas muito próximas da média de aprovação do Ensino Médio, demonstrando a falta de alguns conhecimentos ou habilidades para a aprovação. Por outro lado, chama-se atenção para os estudantes Aprovados, pois essas disciplinas representam o seu grupo de melhor desempenho, ou seja, demonstra uma tendência, facilidade ou habilidade para lidar com assuntos ligados à área de conhecimento de Exatas. Neste sentido é possível afirmar que estes estudantes desenvolveram na educação básica habilidades necessárias para a resolução de problemas.

\section{Conclusões e trabalhos futuros}

Conclui-se que os estudantes que durante a Educação Básica conseguiram melhores resultados nas disciplinas de Matemática, Física e Português são aqueles que tiveram melhores resultados em Algoritmos e Programação. Isto demonstra que competências desenvolvidas nestas disciplinas auxiliam na aprendizagem de Algoritmos e Programação. Tendo em vista que a competência de resolução de problemas está 
VII Congresso Brasileiro de Informática na Educação (CBIE 2018)

Anais dos Workshops do VII Congresso Brasileiro de Informática na Educação (WCBIE 2018)

diretamente ligada a estas áreas de conhecimento, onde habilidades como abstração, interpretação, sequenciamento, raciocínio lógico, entre outras são desenvolvidas.

Com relação aos estudantes Reprovados percebe-se que possuem dificuldade para encontrar soluções algorítmicas, isto confirma a citação de Giraffa e Moura (2015, p.3), onde afirmam que "os, estudantes ingressam na IES com deficiências da Educação Básica, como interpretação de textos e enunciados, hábitos de estudo e pesquisa e, especialmente, com formação deficitária no que tange a conteúdos de Matemática".

Um novo modelo de ensino para Educação Básica, a partir da BNCC (Base Nacional Comum Curricular) visa o desenvolvimento de competências na Educação Básica que contribuem para a formação profissional e acadêmica do estudante do século XXI. Dentre elas está a competência que é Cultura Digital, promovendo a inserção do Pensamento Computacional em todas as áreas do conhecimento, logo, acredita-se que a competência de resolução de problemas será melhor desenvolvida, auxiliando na aprendizagem de Algoritmos e Programação.

Como trabalhos futuros propõem-se um modelo de ensino que auxilie no desenvolvimento da competência de resolução de problemas, com base nas atividades do PC plugadas e/ou desplugadas.

\section{Referências}

AMARAL, Érico Marcelo Hoff do. Processo de Ensino e Aprendizagem de Algoritmos integrando ambientes imersivos e o paradigma de blocos de programação visual. Tese UFRGS - PPGIE - Porto Alegre, 2015.

APA: American Psychiatric Association. Manual Diagnóstico e estatístico de transtornos mentais: DSM-5 [recurso eletrônico] ; tradução: Maria Inês Correa Nascimento... et al.]; revisão técnica: Aristides Volpato Cordioli... [et al.]. $5^{\mathrm{a}}$ ed. Porto alegre : Artmed, 2015. Disponível em: <encurtador.com.br/agmQ9>. Acesso em: 14 abr. 2018.

ARAÚJO Ana Liz Souto Oliveira de; ANDRADE, Wilkerson L.; GUERRERO Dalton D. Serey. Um Mapeamento Sistemático sobre a Avaliação do Pensamento Computacional no Brasil. V Congresso Brasileiro de Informática na Educação (CBIE 2016), 1147-1158.

BEHAR, Patrícia Alejandra. Competência em Educação a distância [recurso eletrônico]/Organizadora , Patrícia Alejandra Behar. - Dados Eletrônicos. - Porto Alegre: Penso, 2013.

BORGES, Maria Fernanda; RUBIO, Juliana de Alcântara Silveira. A Educação Psicomotora como instrumento no Processo de Aprendizagem. Revista Eletrônica Saberes da Educação - Volume 4 - nº 1 - 2013.

CARDOSO, Rogério; ANTONELLO, Sérgio Luis. Interdisciplinaridade, programação visual e robótica educacional: relato de experiência sobre o ensino inicial de programação. Anais dos Workshops do IV Congresso Brasileiro de Informática na Educação (CBIE 2015). DOI: 10.5753/cbie.wcbie.2015.1255.

CHEMIN, Beatris Francisca. Manual da Univates para trabalhos acadêmicos: planejamento, elaboração e apresentação / Beatris Francisca Chemin. - 3. ed. -Lajeado: Ed. da Univates, 2015. 
VII Congresso Brasileiro de Informática na Educação (CBIE 2018)

Anais dos Workshops do VII Congresso Brasileiro de Informática na Educação (WCBIE 2018)

FRAGA, Jordana Ovídio; GONÇALVES, Ana Jandira Nascimento. Dificuldade de Aprendizagem. Revista Maiêutica, Indaial, v. 5, n. 01, p. 43-48, 2017.

GIRAFFA, Lucia Maria Martins; MORA, Michael da Costa. Evasão na disciplina de algoritmo e programação: um estudo a partir dos fatores intervenientes na perspectiva do aluno. III Conferencia Latinoamericana sobre el Abandono en la Educación Superior (III CLABES), 2015.

GRAMINHA, S. S. V. (1994a). Problemas emocionais/comportamentais em uma amostra de escolares: Incidência em função do sexo e idade. Psico, 25, 49-74.

HENRIQUE, Mychelline Souto; TEDESCO, Patrícia C. de A. R.. Uma Revisão sistemática da Literatura sobre conhecimentos, habilidades, atitudes e competências desejáveis para auxiliar a aprendizagem de programação. VI Congresso Brasileiro de Informática na Educação (CBIE 2017). Anais dos Workshops do VI Congresso Brasileiro de Informática na Educação (WCBIE 2017).

HOED, Raphael Magalhães. Análise da evasão em cursos superiores: o caso da evasão em cursos superiores da área de Computação /Raphael Magalhães Hoed; orientador Marcelo Ladeira.- Brasília, 2016.

Competência: Revista da Educação Superior do Senac-RS/Serviço Nacional de Aprendizagem Comercial do Rio Grande do Sul. - Vol. 1, n. 1 (dez. 2008) - Porto Alegre: Pallotti, 2008

LIMA, Sedinei J. S. de; SILVA, Eduardo F. da; ALVES, Victor M.; CASTANHO, Carla L. O.; ESPINDOLA Pablo; BACHINSKI, Rodrigo E.. Aplicação de uma Metodologia Ativa para o Ensino de Lógica de Programação. Anais do EATI Encontro Anual de Tecnologia da Informação e STIN - Simpósio de tecnologia da Informação da Região Noroeste do RS. Ano 6 n. 1 p. 209-212, 2016.

MARTURANO, E. M., Linhares, M. B. M. \& Parreira, V. L. C. (1993). Problemas emocionais e comportamentais associados a dificuldades na aprendizagem escolar. Medicina, 26, 161-175.

KOSCIANSKI,André; GLIZT, Fabiana Rodrigues de Oliveira. O Pensamento Computacional nos anos iniciais do Ensino Fundamental. Revista Novas Tecnologias na Educação. CINTED-UFRGS, V. 15 Nº 2, dezembro, 2017.

PERRENOUD, Philippe. Avaliação da excelência à regulação das aprendizagens: entre duas lógicas. Porto Alegre: Artmed, 1999.

PERRENOUD, Philippe. Dez novas competências para ensinar. Artmed, 2000.

PERRENOUD, Philippe. Porquê construir competências a partir da escola ? Desenvolvimento da autonomia e luta contra as desigualdades. Porto : ASA Editores, 2001.

POLYA, George. (1995). A arte de resolver problemas. Rio de Janeiro: Interciencia.

SILVA, Nyara; LIMA, Ana Carina; SOUZA, Niellen; SOUZA, Decíola. Raciocínio Lógico nas Escolas: Uma Introdução ao Ensino de Algoritmos de Programação. VI Congresso Brasileiro de Informática na Educação (CBIE 2017). Anais dos Workshops do VI Congresso Brasileiro de Informática na Educação (WCBIE 2017).

WING, J. 2006. Computational thinking. Commun. ACM, 49. p.3335 